\title{
Seasonality of stroke: winter admissions and mortality excess
}

\section{A Thailand National Stroke Population Database Study}

By Nicole Lorking BSc ${ }^{1^{*}}$, Adrian D Wood $\mathrm{PhD}^{1^{*}}$, Somsak Tiamkao MD ${ }^{2,3}$, Allan B Clark $\mathrm{PhD}^{4}$, Kannikar Kongbunkiat $\mathrm{MD}^{3,5}$, Joao H Bettencourt-Silva $\mathrm{PhD}^{6}$, Kittisak Sawanyawisuth $\mathrm{MD}^{5}$, Narongrit Kasemsap $\mathrm{MD}^{3,5}$, Mamas A. Mamas DPhil ${ }^{7}$, Phyo K Myint MD ${ }^{1}$.

${ }^{1}$ Ageing Clinical and Experimental Research Group, Institute of Applied Health Sciences, School of Medicine, Medical Sciences \& Nutrition, University of Aberdeen, Aberdeen, AB252ZD, UK.

${ }^{2}$ Neurology Division, Department of Medicine, Faculty of Medicine, Khon Kaen University, Khon Kaen, Thailand 40002.

${ }^{3}$ North-eastern Stroke Research Group, Khon Kaen University, Khon Kaen, Thailand 40002.

${ }^{4}$ Norwich Medical School, University of East Anglia, Norwich, NR47TJ, UK.

${ }^{5}$ Ambulatory Medicine Division, Department of Medicine, Faculty of Medicine, Khon Kaen University, Khon Kaen, Thailand 40002.

${ }^{6}$ Clinical Informatics, Department of Medicine, University of Cambridge, Cambridge CB20QQ UK.

${ }^{7}$ Keele Cardiovascular Research Group, Centre for Prognosis Research, Institute for Primary Care and Health Sciences, Keele University, Stoke-on-Trent, ST55BG, UK.

*Authors Lorking and Wood contributed equally as joint first authors.

Running title: Mortality excess in winter stroke in Thailand

Keywords: Stroke, Seasonality, Mortality excess, Thailand, Cohort study.

\section{Correspondence to:}

Professor Phyo Kyaw Myint

Room 4:013, Polwarth Building, School of Medicine, Medical Sciences and Nutrition, Foresterhill, Aberdeen, AB25 2ZD, Scotland, UK.

Tel: +44 (0) 1224437841

Fax: +44 (0) 1224437911

E-mail: phyo.myint@abdn.ac.uk 


\title{
Seasonality of stroke: winter admissions and mortality excess \\ A Thailand National Stroke Population Database Study
}

\begin{abstract}
Objectives: We examined the existence and potential burden of seasonality of stroke admissions and mortality within a tropical climate using cohort data collected between 1st November 2003 and 31st October 2012.

Patients and methods: In a prospective cohort of hospitalised stroke patients from the catchment of $\sim 75 \%$ of the Thai population $(n=569,307$; mean SD age $=64(14.5))$, incident stroke admissions, inhospital mortality, prolonged hospitalisations, and stroke related complications by season were determined. Rates of incident stroke admissions by month and season were plotted. Winter excess indexes for study outcomes expressed as a percentage were calculated. Using logistic regression we examined the association between winter admission and in-hospital mortality (non-winter admission as reference) adjusting for age, sex, stroke type, year of admission, and presence of pre-existing comorbidities.
\end{abstract}

Results: We observed a winter excess in mortality during hospitalisation (+10.3\%) and prolonged length of stay (+7.3\%). Respective winter excess indexes for dyslipidaemias, arrhythmias, anaemia, and alcohol related disorders in patients that died during hospitalisation were $+1.4 \%,+6.2 \%,+0.2 \%$, $+1.5 \%$. In these patients, respective winter excess indexes for post-stroke complications of pneumonia and sepsis were $+6.7 \%$ and $+3.2 \%$. In fully adjusted analyses, winter admission (compared to non-winter admission) was associated with increased odds of in-hospital mortality (OR $(95 \% \mathrm{Cl})=1.023(1.006-1.040))$.

Conclusions: We provide robust evidence for the existence of an excess in winter stroke admissions and subsequent in-hospital deaths within a tropical region.

Keywords: Stroke, Seasonality, Mortality excess, Thailand, Cohort study. 


\section{Introduction}

Stroke presents a profound challenge to healthcare systems worldwide. The absolute number of people affected by, or who remain disabled from stroke has increased globally from 1990-2013 in both men and women of all ages (1). This burden appears to be compounded an excess in winter hospital admissions and subsequent deaths due to stroke in both temperate geographical regions (2-8) and in subtropical regions (9-14). Some investigators have reported evidence of a winter excess for haemorrhagic stroke only (9-13). Others have shown a winter excess in stroke independent of pathophysiology (14), or found no association between stroke and season (15-17). Previous studies may be limited by small sample size, single centre design, and inability to control for or consider important co-morbid factors.

Delineating the factors contributing to seasonal excess in hospital admissions and deaths in persons with stroke may ultimately help to reduce the overall burden of this pathology. There is a paucity of data on the existence of seasonality in hospital admissions and deaths from stroke within tropical climates, where seasonal patterns are comparably less variable than those observed in subtropical and temperate regions. Indeed, in Thailand the winter period is different than in the subtropical or polar regions with weather conditions that are generally hot and humid year-round and only modest seasonal variation in temperature. There are also limited data on the existence of seasonal variation of comorbidities and common and important post-stroke complications in persons with stroke from tropical regions. Determining the presence of seasonality within such variables would have important implications for improving future clinical outcomes of stroke within this population. We have therefore employed an observational epidemiological approach to examine the seasonality of hospital stroke admissions in a tropical region. Our objectives were to:

a) Determine incident admissions, in-hospital mortality, prolonged hospitalisations, and stroke related complications by season in a prospective cohort of hospitalised stroke patients from 
the catchment of $\sim 75 \%$ of the Thai population followed up between November 2003 and October 2012.

b) Calculate winter excess indexes expressed as a percentage for the above study outcomes.

c) Plot rates of incident stroke admissions and outside air temperature by month and season across study follow up.

d) Determine the association between winter admission (compared with non-winter admission) and in-hospital mortality adjusting for patient demographics, stroke related complications, and pre-existing comorbidities.

\section{Materials and Methods}

Participants and study design

Our study utilised routine consecutive admission data representative of all hospital admissions in Thailand. Data were examined from a cohort of 569,307 prospectively identified participants (mean [SD] age $=64[14.5]$ years) admitted to large and smaller provincial Thai hospitals between $01^{\text {st }}$ November 2003 and $31^{\text {st }}$ October 2012 with a diagnosis of stroke. Details of this National Stroke Database have been published previously (18-19). Briefly, data were obtained from the Universal Coverage Health Security Insurance Scheme Database in Thailand. The Thai population is covered by 3 insurance schemes. The Civil Servant Benefit System covers government employees and their dependents ( $\approx 7 \%$ of the population), and the Social Security scheme covers private sector employees ( $\approx 13 \%$ of the population). The Universal Coverage Health Security Scheme is a basic health insurance scheme covering the majority of the remaining population. Importantly therefore, the 'incident stroke admissions' examined in this whole of population study represents incident cases only in those persons who are registered with the Universal Coverage Health Security Insurance Scheme (approximately $80 \%$ of the total population of Thailand). Additionally, whilst the location of each admission is not available within the current database, with the vast population coverage offered by this scheme, it is likely that regional admission demographics would follow 
trends in population distribution and density, with Central Thailand being the most densely populated of the four regions (20).

In Thailand, diagnosis of stroke is made during individuals' inpatient hospital stay by attending clinical teams based on clinical features and investigation findings, including brain imaging (since 2009, all patients with suspected stroke receive a head computed tomographic scan). All hospitalized patients were clinically managed in accordance with Thai National Clinical Practice Guidelines. Demographic and clinical data were obtained from insurance reimbursement forms using International Classification of Disease, Tenth Revision (ICD-10) codes on an annual basis. Stroke types were categorized as haemorrhagic $(161,162)$, ischaemic (163), or stroke of undetermined pathology (164). Comorbid conditions and stroke related complications were similarly identified. For this study, we collected information on the following comorbid conditions: rheumatic mitral valve disease (105), hypertension (110), ischaemic heart disease (125), atrial fibrillation and flutter (148), heart failure (150), anaemias (D50, D53, D56, D58, D59), dyslipidaemias (E78, E11), alcohol related disorders (F10), neurological disorders (G31, G40, G81), chronic obstructive pulmonary disease (J44) and chronic kidney disease (N18). Serious and common post-stroke complications examined in the present study were pneumonia $(J 14, J 15, J 18)$, respiratory failure $(J 96)$, sepsis (A40, A41), convulsions (R56), myocardial infarction (I21), and shock (R57). Our study protocol conforms to the ethical guidelines of the 1975 Declaration of Helsinki. Ethical approval was obtained from the Ethics Committee in Human Research, Khon Kaen University, Khon Kaen, Thailand.

\section{Study outcomes}

Incident stroke rates (per 100,000) were calculated for each month and season across follow up. Data on the population of Thailand from 2003-2012 for calculating incident stroke rates were obtained from DataBank World Development Indicators (20). Admission data were categorised into three seasons in accordance with annual weather trends detailed by the Meteorology Department of Thailand (21). Winter was defined as November to February, the hot season from March to June, 
and the rainy season from July to October. Primary study outcomes were number of hospital admissions, mortality status at hospital discharge, prolonged (longer than median) length of hospitalisation, and occurrence of serious and common stroke related complications (pneumonia, respiratory failure, sepsis, shock, seizures, and myocardial infarction). Length of hospitalisation was calculated as the difference between date of admission and discharge. Prolonged length of hospitalisation was defined as greater than median length of stay (14 days).

\section{Statistical analysis}

Analyses were conducted using SPSS for Windows version 24.0 (SPSS Inc. Chicago, IL). Descriptive data were obtained for all patients. Chi-squared analyses were employed to compare patient characteristics by season (winter vs. hot and winter vs. rainy seasons). For each study variable (sex, stroke type, comorbidities, post-stroke complications, prolonged length of hospitalisation, and mortality) the winter excess index expressed as a percentage was calculated using Curwen's method as follows: winter admissions - 0.5(admissions in hot season + admissions in rainy season $) /(0.5$ (admissions in hot season + admissions in rainy season $))^{*} 100(22)$. Monthly and seasonal rates of admission per 100,000 population were calculated and plotted across study follow up. Historical data on monthly mean temperatures in Thailand obtained from The World Bank (23) were also plotted across study follow up. For patients that died during acute hospital admission, the winter excess index was calculated for sex, stroke type, comorbidities, and post-stroke complications with comparison by season (winter vs. hot and winter vs. rainy seasons) examined using chi-squared analyses.

The association between winter admission (compared with non-winter admission as the reference category) and in-hospital mortality was examined using logistic regression. To minimise confounding we selected covariates according to current literature and clinical judgement as potentially important for patient prognosis following stroke. The unadjusted association was initially determined (model A), with subsequent additional adjustment for age, sex, stroke type, year of 
admission and pre-existing hypertension, dyslipidaemia, chronic kidney disease, arrhythmias, valvular heart disease, chronic ischaemic heart disease, anaemia, chronic obstructive pulmonary disease, endocarditis, hemiplegia and hemiparesis, and alcohol related disorders (model B). Analyses were adjusted for number of days in each season.

In supplemental analyses, data were stratified into quartiles of age and the winter excess index was calculated for study outcomes of incident hospital admissions, prolonged length of hospitalisation, and mortality (with chi-square analyses to compare outcomes by season (winter vs. hot and winter vs. rainy seasons)). Separate analyses were conducted for men and women. Predictive factors for in-hospital mortality during winter (with non-winter deaths as reference) were explored using multivariable logistic regression.

\section{Results}

In total, 569,307 patients (mean [SD] age $=64$ [14.5]) were included in this study (55\% men and $45 \%$ women), with diagnoses of ischaemic $(51.3 \%)$, haemorrhagic (32.9\%) or undetermined (15.8\%) stroke. Table 1 shows the characteristics of study participants by season. In all patients, there was a winter excess for haemorrhagic $(+17.6 \%)$, and undetermined $(+4.9 \%)$ stroke types, prolonged length of hospitalisation (+7.3\%), and acute mortality $(+10.3 \%)$. Women were older $(65.7$ (14.5) than men (62.2 (14.4). The proportion of patients with a history of rheumatic mitral valve disease was greater in patients admitted during winter months compared to non-winter months. Pre-existing hypertension, dyslipidaemias, anaemia, arrhythmias, and chronic ischaemic heart disease were more prevalent in patients admitted in winter compared to the hot season. Stroke related complications of pneumonia and sepsis were more prevalent in winter months.

Table 2 shows sex, stroke type, history of comorbidities, and presence of stroke related complications in patients that died during hospitalisation (with corresponding winter excess indices). Results show higher total winter deaths compared to the rainy season for both men (winter excess index $=+12.4 \%$ ) and women (winter excess index $=+7.8 \%$ ). Number of stroke deaths in winter were 
higher for all stroke subtypes. Patients that died during winter were more likely to have a history of dyslipidaemias (winter excess index $=+1.4 \%$ ) compared to non-winter months, whilst those with pre-existing chronic kidney disease and arrhythmias were more likely to die in winter compared to the hot season (winter indices: $+1.7 \% ;+6.2 \%$ respectively). Patients that died in winter were also more likely to suffer from stroke related complications of pneumonia (winter excess index $=+6.7 \%$ ), and sepsis (winter excess index $=+3.2 \%$ ). Those with respiratory failure were more likely to die in winter compared to the hot season.

Rates of incident stroke admissions by month (November 2003 - October 2012) and season (winter season 2003/4 to rainy season 2012) are shown in Figures 1 and 2 respectively (with full data presented in Supplementary Tables $\mathbf{1}$ and 2). Stroke admission rates increased annually across the entire study follow up period. Rates of admission (n (rate/100,000)) during winter, hot, and rainy seasons respectively in 2003/4 were 16387(31.5), 13684(26.31), 14967(28.78) and in 2012/13 were 28862 (53.18), 27678 (51.00), and 29260 (53.91) in 2012). In fully adjusted analyses, winter admission (compared to non-winter admission) was associated with increased odds of in-hospital mortality (OR $(95 \% \mathrm{Cl})=1.023(1.006-1.040))($ Table 3) .

\section{Supplementary analyses}

Results of supplementary analyses are shown in Supplementary Tables 3-5. In men, the number of hospital admissions was higher in winter compared to the hot season for all stroke types, particularly in older participants. There was no difference in stroke admissions between the winter and rainy seasons (Supplementary Table 3). Similar results for acute admissions were observed in women (Supplementary Table 4). Prolonged length of hospitalisation in winter was prominent following haemorrhagic stroke in older participants. In men over 75 admitted during winter with haemorrhagic stroke, $14.2 \%$ had greater than median length of hospitalisation (compared with $13.1 \%$ and $13.9 \%$ during hot and rainy seasons respectively; winter excess index $=24.7 \%)$. Mortality in older men (aged $>75$ years) with ischaemic stroke was higher in winter compared with non-winter 
( $27.3 \%$ vs. $25.3 \%$ and $26.7 \%$ for hot and rainy seasons respectively; winter excess index $=+19.9 \%$ ).

Similar associations in men were found for haemorrhagic (winter vs. hot season) and undetermined stroke subtypes (winter vs. non-winter). Mortality was higher in older women (> 65 years) with ischaemic stroke in winter compared to the rainy season ( $28.9 \%$ vs. $28.2 \%$ respectively for $66-75$ years category (winter excess index $=+13.9 \%$ ) and $41.2 \%$ vs. $40.6 \%$ for $>75$ years category (winter excess index category $=+21.3 \%)$ ). Multivariable regression showed that older age and haemorrhagic stroke were associated with increased odds for winter death, whereas female sex and more recent year of hospitalisation were associated with reduced odds of winter death (Supplementary Table 5).

\section{Discussion}

We have conducted the largest and most extensive study to date, which examines the seasonality of acute stroke in a tropical country. This study shows a winter excess in acute stroke admissions, length of hospitalisation, and inpatient mortality for patients with stroke admitted to hospital in Thailand between November 2003 and October 2012. Mortality excess during the winter months is independent of patient characteristics, comorbidities, and stroke related complications. The presence of excess stroke admissions and in-hospital mortality during winter provides evidence that this phenomenon is not only restricted to developed countries with temperate climates. Stroke related complications of pneumonia and sepsis are more common in the winter months.

Annual seasonal variation in temperature in Thailand is modest. Data collected between 2003 and 2012 shows mean temperatures in Thailand during winter, hot, and rainy seasons respectively of $24.6^{\circ} \mathrm{C}, 28.2^{\circ} \mathrm{C}$, and $27.0^{\circ} \mathrm{C}$ (23) (although variation is greater in the North compared to the South of the country) (21). The majority of previous studies conducted in subtropical regions examining seasonal variation in stroke do not report on temperature variation across seasons (9$13,15,17)$. In those that do $(14,16)$, mean differences between hot and cold seasons are substantially greater than in Thailand, although the extent of excess winter mortality is comparable with our 
observations (14). Relative temperature change may therefore be more strongly related to stroke occurrence and mortality rather than absolute mean differences in temperature. Indeed, our data show that hospital admissions tend to peak around November to December (Figure 1) which coincides with initial temperature drop during winter. Admissions then fall abruptly in January to February, thus it is possible that the initial effect of temperature dropping may explain the increase in stroke admissions during winter.

Older people, especially those above 75 years of age, were more likely to be admitted during winter, have a longer hospital stay, and increased likelihood of winter death compared to younger patients. These findings support previous research examining the seasonality of cerebrovascular disease, which proposes that older people demonstrate an exaggerated physiological response in colder conditions, such as a larger increase in blood pressure and propensity for coagulability (2427). People living in temperate geographical regions may be less likely to have adequate protection against cold exposure, such as heated housing and protective outdoor clothing (28). It is plausible that such factors could contribute to an exaggerated physiological response to colder conditions in the elderly population. Prolonged length of hospitalisation in older participants may relate to increased likelihood of greater complex care needs and rehabilitation requirements in these individuals (29).

It has previously been shown that blood pressure follows a seasonal pattern (30) and an inverse association between outside air temperature and blood pressure measurements has been described in several populations (31-34). Previous history of hypertension or blood pressure of 140/90 mm Hg or higher is a well established independent risk factor for acute stroke (35). Our observed increase in winter stroke admissions for patients with hypertension and chronic kidney disease compared to the hot season supports the notion that elevated blood pressure may be more likely to cause harm in colder conditions.

Patients with dyslipidaemias, including type II diabetes mellitus, were more likely to be admitted in winter and have higher winter mortality compared to other seasons. It is unclear how 
dyslipidaemia could contribute to the winter excess in admissions and mortality observed in our study, although lower outside air temperatures have previously been associated with an unfavourable lipid profile. In a South Korean study of 55,567 participants examining the association between temperature and conventional cardiovascular risk markers over 13 years, every $10^{\circ} \mathrm{C}$ decrease in the minimum temperature was associated with a mean (SD) increase in LDL cholesterol of $0.08(0.04) \mathrm{mg} / \mathrm{dL}$ and a reduction in $\mathrm{HDL}$ cholesterol of $0.05(0.02) \mathrm{mg} / \mathrm{dL}$ (33). Higher winter mortality in patients with arrhythmias reflects a higher risk for cardioembolic stroke in these individuals, with stasis of blood flow elevating the risk for arterial thromboembolism (36-37). Regression analysis showed that admission to hospital during the winter months was associated with increased odds of in-hospital mortality. Although this association was statistically significant, we would exercise cautious interpretation of the results. Whilst the large populationbased study design which we employed has many advantages, this approach is prone to finding many associations even if they are small and not necessarily clinically meaningful.

Our study has a number of strengths. We have conducted the largest study to date that examines winter excess in stroke admissions and clinical outcomes in a tropical region. An observational epidemiology approach was utilised to address our research objectives using routine consecutive admission data representative of all hospital admissions in Thailand. We have conducted a nationwide analysis and are therefore powered to detect small effect sizes as well as subtle changes in incident stroke admissions. Our study included data from multiple centres over a timespan of ten years, and employed a winter excess index calculation as a method of obtaining further information about the effect of variables such as stroke type, sex, and age on stroke seasonality. The seasonality of comorbidities and post-stroke complications as well as their influence on winter mortality for each stroke subtype was also examined, providing insight into their contribution towards stroke outcome.

We acknowledge some limitations. Incident stroke admission cases examined in this study were obtained from The Thailand Universal Coverage Health Security Insurance Scheme Database 
which covers approximately $80 \%$ of the population. Thus, our data does not capture persons who are insured by the Civil Servant Benefits System or the Social Security Scheme (remaining $20 \%$ of the population). This health Insurance Scheme database did not provide information on lifestyle or social factors, the clinical severity of the strokes recorded, or medication use prior to stroke admission. Such data which may have allowed further insight into the observed winter excess in acute stroke mortality. It also omits milder cases of stroke that were not admitted to hospital, in addition to severe cases in which patients died prior to admission. Indeed, prior to 2008, current Thai nationwide clinical practice guidelines for the management of stroke were only employed in provincial or community hospitals, which may have an influence on our measurement of stroke incidence prior to this time. In 2008 however the Ministry of Public Health (MoPH) and the National Health Security Office (NHSO) implemented the Stroke Fast Track Programme to all levels of hospital in Thailand (38). It should be noted that when observing the overall epidemiological trend in stroke admission rates (Figure 1), there is no noticeable change in the trajectory of stroke incidence following the implementation of this policy, providing support for our measurement of stroke admission rates prior to 2008. Importantly, it is likely that we are now detecting and treating more stroke cases after implementation of the Stroke Fast Track Programme rather than an increase in stroke incidence across the duration of the study. The sensitivity and specificity of the algorithm for identifying stroke cases in the Thailand Universal Coverage Health Security Insurance Scheme Database is uncertain. It has previously been shown in a population based Canadian cohort that hospital administrative data are a reliable means of reporting ICD-10 coded ischaemic stroke and intracerebral haemorrhage (39).

The Thai Ministry of Public Health divides Thailand into 13 geographical areas with approximately 5 million persons and 5,000 hospital per area. It is not clear whether our findings are similar across all hospitals in Thailand or are influenced by seasonal findings in a subset of hospitals. It is possible that incidence of stroke hospitalisation at different times of year may be influenced by severity of stroke. In Thailand, Facial drooping, Arm weakness, Speech difficulties and Time to call 
emergency services (FAST) has been employed to enhance public awareness for the early detection of stroke. Persons who seek medical attention who have suspected stroke are assessed using FAST screening and admitted directly to provincial or large community hospitals, (some of which have specialised centres for the management and treatment of acute stroke) after initial assessment in the community, emergency department or outpatient setting. Importantly, all patients with suspected stroke in Thailand must be referred to hospital for definite diagnosis and stroke services in Thailand are the same standard across the country. Brain imaging is performed urgently and patients are managed in line with contemporary stroke care guidelines (38).

Starting the study in November 2003 and ending in October 2012 may lead to small biases in the context of a major temporal trend in incident stroke admissions. Additionally, whilst patient follow-up data is limited due to data being collected during acute hospital admission, the aim of the current study was to investigate the presence or absence of a winter excess in stroke admission and mortality in a tropical region. We did not examine the association between monthly temperature recordings and stroke admissions and mortality for each year between 2003 and 2012, although adjustment for the effect of year on winter death was made in our analyses. We did not however account for variability adjudicated to the year in terms of seasons nested within each year. Further, we acknowledge that the use of population based ecological data does not allow for inferences to be made on an individual level, however our primary purpose was to examine seasonal trends in stroke admission in a tropical region to inform patients, public, clinicians and service providers.

Whilst collecting data over a time period of nine years increases the likelihood of healthcare system use being subject to significant changes in social and economic developments, the introduction of the Universal Health Security Insurance Scheme in 2002 resulted in nationwide accessible healthcare, with the extensive geographical coverage favouring poorer households and elderly populations $(40,41)$. It is therefore unlikely that percentage population use of such services would have significantly decreased over this time period. 


\section{Summary and Conclusion}

Stroke remains a significant challenge to government resources and healthcare systems in developing countries such as Thailand, with an ageing population serving to increase this demand in the future. Overall, the current study expands upon previous research as the largest study to examine the seasonality of stroke in a tropical country, and provides strong evidence for the presence of a winter excess in admissions, mortality, and length of stay for ischaemic, haemorrhagic, and undetermined stroke subtypes, as found in temperate climates. We have also identified comorbid conditions and complications that are associated with the winter excess phenomenon in a tropical setting, suggesting there may be potential to improve clinical outcomes by targeted case management strategies. In summary, it is vitally important that future research continues to focus on enhancing our understanding of stroke demographics in regions outside of the developed world with the aim of identifying and addressing important prognostic factors contributing to the seasonal burden of this condition.

\section{Acknowledgements}

We wish to thank the administrative staff of Insurance Schemes who prepared the anonymised data sets. We are grateful to Tiberiu A Pana for assistance in generating the figures.

\section{Author contributions}

P.K.M. conceived the study, S.T. co-ordinated the data acquisition and obtained ethical approval, A.B.C. and J.H.B.-S. cleaned the data, P.K.M. formulated analysis plan, N.L. and A.D.W analysed the data, N.L. and A.D.W. drafted the paper. All authors contributed in writing of the paper. P.K.M. is the guarantor.

\section{Sources of funding}


ADW was funded by the Special Educational Scholarship award by the Department of Medicine for the Elderly, NHS Grampian [grant number ES798].

\section{References}

1. Feigin, V. L., Norrving, B., \& Mensah, G. A. Global Burden of Stroke. Circulation Research. 2017: 120:439-448.

2. Zorilla-Vaca, A., Healy, R. J., Silva-Medina, M. M. Revealing the association between cerebrovascular accidents and ambient temperature: A meta-analysis. International Journal of Biometeorology. 2017:5(1): 821-832.

3. Takizawa, S., Shibata, T., Takagi, S., \& Kobayashi, S.: Seasonal variation of stroke incidence in japan for 35631 stroke patients in the Japanese standard stroke registry 1998-2007. Journal of Stroke and Cardiovascular Diseases. 2013: 22(1): 36-41.

4. Ziauddin, I. N., Shah, I. A., Ali, Z., \& Mehmood, K.: Seasonal variation in stroke in a teaching hospital of Khyber Pakhtunkhwa. Journal of Postgraduate Medical Institute. 2015: 29(3): 193-198.

5. Jakovljevic, D., Salomaa, V., Sivenius, J., Tamminen, M., Sarti, C., \& Salmi, K. et al. Seasonal variation in the occurrence of stroke in a Finnish adult population: The FINMONICA stroke register. Stroke. 1996: 27(10): 1774-1779.

6. Khan, F. A., Engstrom, G., Jerntorp, I., Pessah-Rasmussen, H., \& Janzon, L.: Seasonal patterns of incidence and case fatality of stroke in Malmö, Sweden: The STROMA study. Neuroepidemiology. 2005: 24: 26-31.

7. Kelly-Hayes, M., Wolf, P. A., Kase, C. S., Brand, F. N., \& Mcguirck, J. M.: Temporal patterns of stroke onset. The Framingham study. Stroke. 1995: 26(8): 1343-1347.

8. Myint, P.K., Vowler, S. L., Woodhouse, P. R., Redmayne, O., \& Fulcher, R. A.: Winter excess in hospital admissions, in-patient mortality and length of acute hospital stay in stroke: A 
hospital database study over six seasonal years in Norfolk, UK. Neuroepidemiology. 2007: 28(2): 79-85.

9. Chen, Z. Y., Chang, S. F., \& Su, C. L.: Weather and stroke in a subtropical area: Ilan, Taiwan. Stroke. 1995: 26(4): 569-572.

10. Telman, G., Sviri, G., Sprecher, E., Amsalem, Y., \& Avizov, R. Seasonal variation in spontaneous intracerebral haemorrhage in Northern Israel. Chronobiology International. 2017: 1525-6073.

11. Kumar, P., Kumar, A., Pandit, A., Pathak, A., \& Prasad, K.: Seasonal variations in stroke: A study in a hospital in North India. Journal of Stroke. 2015: 17(2): 219-220.

12. Miah, A. H., Sutradhar, S. R., Ahmed, S., Alam, M., Mondol, G. D., \& Khan, N. A. Seasonal variation in types of stroke and its common risk factors. Mymensingh Medical Journal. 2012: 21(1): 13-20.

13. Hannan, M. A., Rahman, M. M., Hague, A., \& Ahmed, H. U.: Stroke: Seasonal variation and association with hypertension. Bangladesh Med Res Council Bulletin. 2001: 27(2): 69-78.

14. Diaz, A., Gerschcovich, E. R., Diaz, A. A., Antia, F., \& Gonorazky, S.: Seasonal variation and trends in stroke hospitalisations and mortality in a South American community hospital. Journal of Stroke and Cerebrovascular Diseases. 2013: 22(7): 66-69.

15. Raj, K., Bhatia, R., Prasad, K., Srivastava, M., \& Singh, M. B.: Seasonal differences and circadian variation in stroke occurrence and stroke subtype. Journal of Stroke and Cerebrovascular Diseases. 2014: 24(1): 10-16.

16. Gomes, J., Damasceno, A., Carrilho, C., Lobo, V., Lopes, H., \& Madede, T.: The effect of season and temperature variation on hospital admissions for incident stroke events in Maputo, Mozambique. Journal of Stroke and Cerebrovascular Diseases. 2013: 23(2): 271277.

17. Woo, J., Kay, R., \& Nicholis, M. G.: Environmental temperature and stroke in a subtropical climate. Neuroepidemiology. 1991: 10: 260-265. 
18. Wood AD, Mannu GS, Clark AB, Tiamkao S, Kongbunkiat K, Bettencourt-Silva JH, Sawanyawisuth K, Kasemsap N, Barlas RS, Mamas M, Myint PK. Rheumatic mitral valve disease is associated with worse outcomes in stroke: a Thailand national database study. Stroke. 2016: 47(11): 2695-2701.

19. Cumming, K, Tiamkao, S, Kongbunkiat, K, Clark, AB, Bettencourt-Silva, JH, Sawanyawisuth, K, Kasemsap, N, Mamas, MA, Seeley, JA, \& Myint, PK. Impact of HIV on inpatient mortality and complications in stroke in Thailand: a National Database Study. Epidemiology and Infection. 2017: 145(6): 1285-1291.

20. DataBank World Development Indicators Thailand. Available from: https://data.worldbank.org/country/thailand [Accessed 02 June 2019].

21. Thai Meteorology Department. Available from: https://www.tmd.go.th/en/index.php. [Accessed 02 June 2019].

22. Curwen M. \& Davies T. Winter mortality, temperature and influenza: has the relationship changed in recent years? Population Trends. 1988: 54:17-20.

23. The World Bank. Average monthly temperature and rainfall for Thailand from 1901-2015. Available from: http://sdwebx.worldbank.org/climateportal/index.cfm?page=country historical climate\&Th isCCode=THA [Accessed 02 June 2019].

24. Woodhouse, P., Khaw, K., Plummer, M., Meade, T. \& Foley, A.: Seasonal variations of plasma fibrinogen and factor VII activity in the elderly: Winter infections and death from cardiovascular disease. The Lancet. 1994: 343(8895): 435-439.

25. Collins, K., Easton, J., Exton-Smith, A., \& Pluck, P.: Effect of age on body temperature and blood pressure in cold environments. Clinical Science. 1985: 69: 465-470.

26. Pan, W., Li, L., \& Tsai, M.: Temperature extremes and mortality from coronary heart disease and cerebral infarction in elderly Chinese. The Lancet. 1995: 345: 353-355. 
27. Woodhouse, P., Khaw, K., \& Plummer, M.: Seasonal variation of blood pressure and its relationship to ambient temperature in an elderly population. Journal of Hypertension. 1993: 11: $1267-1274$.

28. Somrongthong, R., Dullyaperadis, S., Wulff, A. \& Ward, P.: The effects of housing on health and health risks in an ageing population: A qualitative study in rural Thailand. Biomed Research International. 2014: 1-7.

29. Gaye, B., Canonico, M., Perier, M., Berr, C., \& Elbaz, A.: Ideal cardiovascular health is inversely associated with incident coronary heart disease and stroke events in noninstitutionalised elderly subjects: The three cities study. Circulation. 2016: 134: 16496.

30. Brennan, PJ., Greenberg, G., Miall, WE., Thompson, SG.: Seasonal variation in arterial blood pressure. British Medical Journal. 1982: 285: 919-923.

31. Alpe'rovitch A, Lacombe JM, Hanon O, Dartigues JF, Ritchie K, Ducimetie 're P, Tzourio C. Relationship between blood pressure and outdoor temperature in a large sample of elderly individuals: the Three-City study. Archives of Internal Medicine. 2009: 169:75-80.

32. Kunes J, Tremblay J, Bellavance F, Hamet P. Influence of environmental temperature on the blood pressure of hypertensive patients in Montreal. American Journal of Hypertension. 1991: 4:422-426.

33. Hong, Y. C., Kim, H., Oh, S.Y., Lim, Y. H., Kim, S. Y \& Yoon, H. J: Association of cold ambient temperature and cardiovascular markers. Science of the Total Environment. 2012: 435: 7479.

34. Sartini, C., Barry, S., Whincup, P., Lowe, G., Lennon, L, \& Walsh, P.: Relationship between outdoor temperature and cardiovascular disease risk factors in older people. European Journal of Preventative Cardiology. 2017: 24(4): 349-356.

35. O’Donnell, M.J., Lim Chin, S., Rangarajan, S. et al. Global and regional effects of potentially modifiable risk factors associated with acute stroke in 32 countries (INTERSTROKE): A casecontrol study. Lancet. 2016. 388(10046): p. 761-775. 
36. Ferrieri P; Jones Criteria Working Group. Proceedings of the Jones Criteria workshop. Circulation. 2002: 106: 2521-2523.

37. Violi, F., \& Loffredo, L.: Thromboembolism or atherothromboembolism in atrial fibrillation? Circulation. 2012: 5: 1053- 1055.

38. Suwanwela, N.C. Stroke Epidemiology in Thailand. Journal of Stroke: 2014: 16(1): 1-7.

39. Porter, J., Mondor, L., Kapral M.K., Fang, J., Hall, R.E. How reliable are administrative data for capturing stroke patient and their care. Cerebrovasc Dis Extra. 2016; 6(3): 96-106.

40. Limwattananon S, Tangcharoensathien V, Tisayaticom K, Boonyapaisarncharoen T, Prakongsai P. Why has the Universal Coverage Scheme in Thailand achieved a pro-poor public subsidy for health care? BMC Public Health. 2012;12 Suppl 1:S6.

41. Limwattananon S, Neelsen S, O’Donnell O, Prakongsai P, Tangcharoensathien V, van Doorslaer E, et al. Universal coverage with supply-side reform: the impact on medical expenditure risk and utilization in Thailand. Journal of Public Economics. 2015: 121:79-94.

\section{Figure legends}

Figure 1. Incident stroke rate by month ( $($ rate $/ 100,000)$ ) and mean monthly temperature (oC) (October 2003 - January 2013).

January data for each year falls within vertical lines.

Figure 2. Incident stroke rate by season ( $($ rate/100,000)) and mean seasonal temperature (oC) (winter season 2003/4 - rainy season 2012).

Winter data for each year falls within vertical lines. 
Table 1. Participant characteristics by season with corresponding winter excess index.

\begin{tabular}{|c|c|c|c|c|c|c|c|}
\hline & All** & Winter & Hot & $\begin{array}{c}P \\
\text { (vs winter) }\end{array}$ & Rainy & $\begin{array}{c}P \\
\text { (vs winter) }\end{array}$ & $\begin{array}{c}\text { Winter Excess } \\
\text { Index, \% }\end{array}$ \\
\hline Age, years* & $63.7(14.5)$ & $63.9(14.5)$ & $63.5(14.5)$ & $<0.001$ & $63.9(14.5)$ & 0.04 & - \\
\hline \multicolumn{8}{|l|}{ Sex } \\
\hline Men & 313085 (55) & 107180 (54.9) & 101179 (55.6) & \multirow[t]{2}{*}{$<0.001$} & 104726 (54.5) & \multirow[t]{2}{*}{0.002} & +4.1 \\
\hline Women & $256222(45)$ & $87878(45.1)$ & $80843(44.4)$ & & $87501(45.5)$ & & +4.4 \\
\hline \multicolumn{8}{|l|}{ Stroke type } \\
\hline Ischaemic & $291917(51.3)$ & $94725(32.4)$ & $95426(32.7)$ & \multirow{3}{*}{$<0.001$} & $101766(34.9)$ & \multirow{3}{*}{$<0.001$} & -3.9 \\
\hline Haemorrhagic & $187489(32.9)$ & $69407(37.0)$ & $56609(30.2)$ & & $61473(32.8)$ & & +17.6 \\
\hline Undetermined & $89901(15.8)$ & $30926(34.4)$ & $29987(33.4)$ & & $28988(32.2)$ & & +4.9 \\
\hline \multicolumn{8}{|l|}{ Comorbidities } \\
\hline Dyslipidemias & $111344(19.6)$ & $36215(32.5)$ & $35743(32.1)$ & $<0.001$ & 39386 (35.4) & $<0.001$ & -3.6 \\
\hline Neurological disorders & $52634(9.2)$ & $17843(34.1)$ & $16633(31.8)$ & 0.33 & $17888(34.2)$ & 0.22 & +3.4 \\
\hline Hypertension & $253215(31.6)$ & $86430(34.1)$ & $79932(31.6)$ & 0.007 & $86853(34.3)$ & 0.72 & +3.6 \\
\hline Chronic kidney disease & $17840(3.1)$ & $5917(33.2)$ & $5619(31.5)$ & 0.17 & $6355(35.3)$ & $<0.001$ & -1.2 \\
\hline Arrhythmias & $2493(0.4)$ & $817(32.7)$ & $817(32.7)$ & $<0.001$ & $862(34.5)$ & 0.08 & -2.7 \\
\hline Rheumatic mitral valve disease & $5256(0.9)$ & $1984(37.7)$ & $1676(31.9)$ & 0.001 & $1596(30.4)$ & $<0.001$ & +21.3 \\
\hline Chronic ischaemic heart disease & $15537(2.7)$ & $5085(32.7)$ & $5060(32.6)$ & 0.001 & $5392(34.7)$ & $<0.001$ & -2.7 \\
\hline Anaemias & $30821(5.4)$ & $10234(33.2)$ & 9835 (31.9) & 0.02 & $10752(34.9)$ & $<0.001$ & -0.6 \\
\hline Alcohol related disorders & $8816(1.5)$ & 3074 (34.9) & $2865(32.5)$ & 0.49 & $2877(32.6)$ & 0.02 & +7.1 \\
\hline COPD & 10998 (1.9) & 3834 (34.9) & 3467 (31.5) & 0.09 & 3697 (33.6) & 0.17 & +7.0 \\
\hline Post stroke complications & & & & & & & \\
\hline
\end{tabular}




\begin{tabular}{|c|c|c|c|c|c|c|c|}
\hline Pneumonia & $54368(9.5)$ & 18990 (34.9) & $16704(30.7)$ & $<0.001$ & $18674(34.3)$ & 0.42 & +7.4 \\
\hline Respiratory failure & 39707 (7.0) & 13266 (33.4) & 12425 (31.3) & 0.38 & 14016 (35.3) & $<0.001$ & +0.3 \\
\hline Sepsis & $18541(3.3)$ & $6260(33.8)$ & 6095 (32.9) & 0.008 & $6168(33.4)$ & 0.44 & +2.1 \\
\hline Convulsions & $11942(2.1)$ & 3963 (33.2) & 3738 (31.3) & 0.32 & 4241 (35.5) & $<0.001$ & -0.7 \\
\hline MI & $3260(0.6)$ & $1088(33.4)$ & $1036(31.8)$ & 0.90 & $1136(34.8)$ & 0.09 & +0.2 \\
\hline Shock & $4120(0.7)$ & $1246(30.2)$ & $1276(31.0)$ & 0.01 & $1598(38.8)$ & $<0.001$ & -13.3 \\
\hline \multicolumn{8}{|l|}{ Length of stay } \\
\hline$>14$ days & $5310(9.3)$ & 18548 (34.9) & $16514(31.1)$ & $<0.001$ & $18048(34.0)$ & 0.10 & +7.3 \\
\hline Mortality & 67491 (11.9) & 23990 (35.5) & $21108(31.3)$ & $<0.001$ & $22393(33.2)$ & $<0.001$ & +10.3 \\
\hline
\end{tabular}

*Mean (SD). All other variables presented as $\mathrm{n}(\%)$.

**Shows percentage within all patients included in the study. Remaining columns show percentage of that stratum of patients in the three seasons. 
Table 2. Sex, stroke type, comorbidity and stroke related complication profile in patients that died during acute hospital stay by season, with corresponding winter excess indices.

\begin{tabular}{|c|c|c|c|c|c|c|c|}
\hline & All** & Winter & Hot & $P$ & Rainy & $P$ & $\begin{array}{c}\text { Winter Excess } \\
\text { Index, \% }\end{array}$ \\
\hline Age, years* & $63.8(15.2)$ & $63.8(15.2)$ & $63.5(14.5)$ & 0.001 & $63.9(14.5)$ & 0.02 & - \\
\hline \multicolumn{8}{|l|}{ Sex } \\
\hline $\begin{array}{l}\text { Men } \\
\text { Women }\end{array}$ & $\begin{array}{l}37099(55.0) \\
30392(45.0) \\
\end{array}$ & $\begin{array}{l}13346(55.6) \\
10644(44.4) \\
\end{array}$ & $\begin{array}{l}11571(54.8) \\
9537(45.2) \\
\end{array}$ & 0.04 & $\begin{array}{l}12182(54.4) \\
10211(45.6) \\
\end{array}$ & 0.004 & $\begin{array}{r}+12.4 \\
+7.8 \\
\end{array}$ \\
\hline \multicolumn{8}{|l|}{ Stroke type } \\
\hline Ischaemic & $20571(30.5)$ & $6923(33.7)$ & $6718(32.7)$ & & $6930(33.7)$ & & +1.5 \\
\hline Haemorrhagic & 43876 (65.0) & $15958(36.4)$ & $13398(30.5)$ & $<0.001$ & $14520(33.1)$ & $<0.001$ & +14.3 \\
\hline Undetermined & $3044(4.5)$ & $1109(36.4)$ & $992(32.6)$ & & $943(31.0)$ & & +14.6 \\
\hline \multicolumn{8}{|l|}{ Comorbidities } \\
\hline Dyslipidemias & $4699(7.0)$ & $1581(33.6)$ & 1501 (31.9) & 0.02 & 1617 (34.4) & 0.004 & +1.4 \\
\hline Neurological disorders & $3224(4.8)$ & $1134(35.2)$ & $1036(32.1)$ & 0.19 & 1054 (32.7) & 0.47 & +8.5 \\
\hline Hypertension & 30960 (45.9) & $10986(35.5)$ & $9534(30.8)$ & 0.09 & $10440(33.7)$ & 0.04 & +10.0 \\
\hline Chronic kidney disease & $2809(4.2)$ & $947(33.7)$ & $914(32.5)$ & 0.02 & $948(33.7)$ & 0.06 & +1.7 \\
\hline Arrhythmias & $496(0.7)$ & $172(34.7)$ & $156(31.5)$ & 0.03 & 168 (33.9) & 0.13 & +6.2 \\
\hline Rheumatic mitral valve disease & $546(0.8)$ & $202(37.0)$ & $182(33.3)$ & 0.43 & $162(29.7)$ & 0.08 & +17.4 \\
\hline Chronic ischaemic heart disease & $2098(3.1)$ & $707(33.7)$ & $670(31.9)$ & 0.09 & $721(34.4)$ & 0.05 & +1.7 \\
\hline Anaemias & $5523(8.2)$ & $1843(33.4)$ & $1737(31.5)$ & 0.41 & $1943(35.2)$ & 0.36 & +0.2 \\
\hline Alcohol related disorders & $1467(2.2)$ & $494(33.7)$ & 497 (33.9) & 0.02 & $476(32.4)$ & 0.32 & +1.5 \\
\hline
\end{tabular}




\begin{tabular}{|c|c|c|c|c|c|c|c|}
\hline COPD & $1575(2.3)$ & $573(36.4)$ & $494(31.4)$ & 0.38 & $508(32.3)$ & 0.21 & +14.4 \\
\hline \multicolumn{8}{|c|}{ Post stroke complications } \\
\hline Pneumonia & 15447 (22.9) & $5374(34.8)$ & $4714(30.5)$ & $<0.44$ & $5359(34.7)$ & $<0.001$ & +6.7 \\
\hline Respiratory failure & $16782(24.9)$ & $5464(32.6)$ & $5310(31.6)$ & $<0.001$ & $6008(35.8)$ & $<0.001$ & -3.4 \\
\hline Sepsis & $9668(14.3)$ & $3291(34.0)$ & $3165(32.7)$ & $<0.001$ & $3212(33.2)$ & 0.03 & +3.2 \\
\hline Convulsions & $2045(3.0)$ & $641(31.3)$ & $680(33.3)$ & $<0.001$ & $724(35.4)$ & $<0.001$ & -8.7 \\
\hline $\mathrm{MI}$ & $1228(1.8)$ & 41 (33.9) & $389(31.7)$ & 0.20 & $423(34.4)$ & 0.11 & +2.5 \\
\hline Shock & $2121(3.1)$ & $601(28.3)$ & $672(31.7)$ & $<0.001$ & $848(40.0)$ & 0.98 & -20.9 \\
\hline
\end{tabular}

*Mean (SD). All other variables presented as $\mathrm{n}(\%)$.

**Shows percentage within all patients included in the study. Remaining columns show percentage of that stratum of patients in the three seasons. 
Table 3. Association between winter admission and in-hospital mortality (with non-winter admission as the reference category).

\begin{tabular}{|l|c|c|}
\hline & OR $(95 \% \mathrm{Cl})$ & $P$ \\
\hline Model A & $1.075(1.053-1.093)$ & $<0.001$ \\
\hline Model B & $1.023(1.006-1.040))$ & $=0.008$ \\
\hline
\end{tabular}

Model A: unadjusted

Model B: adjusted for age, sex, stroke type, year of admission, pre-existing hypertension, dyslipidaemia, chronic kidney disease, arrhythmias, valvular heart disease, chronic ischaemic heart disease, anaemia, chronic obstructive pulmonary disease, endocarditis, hemiplegia and hemiparesis, and alcohol related disorders. 


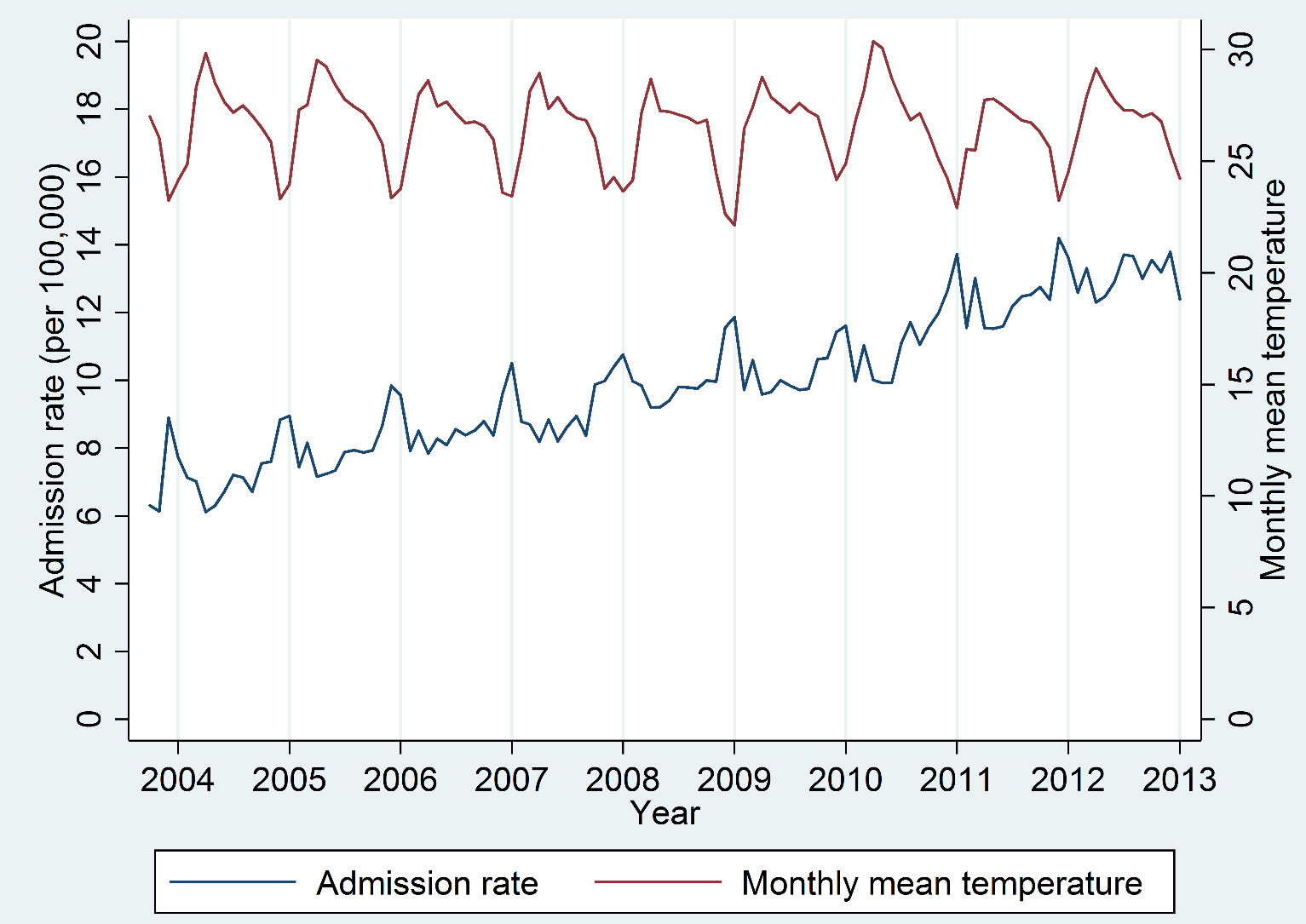

Figure 1. Incident stroke rate by month ( $\mathrm{n}($ rate/100,000)) and mean monthly temperature (oC) (October 2003 - January 2013). January data for each year falls within vertical lines. 


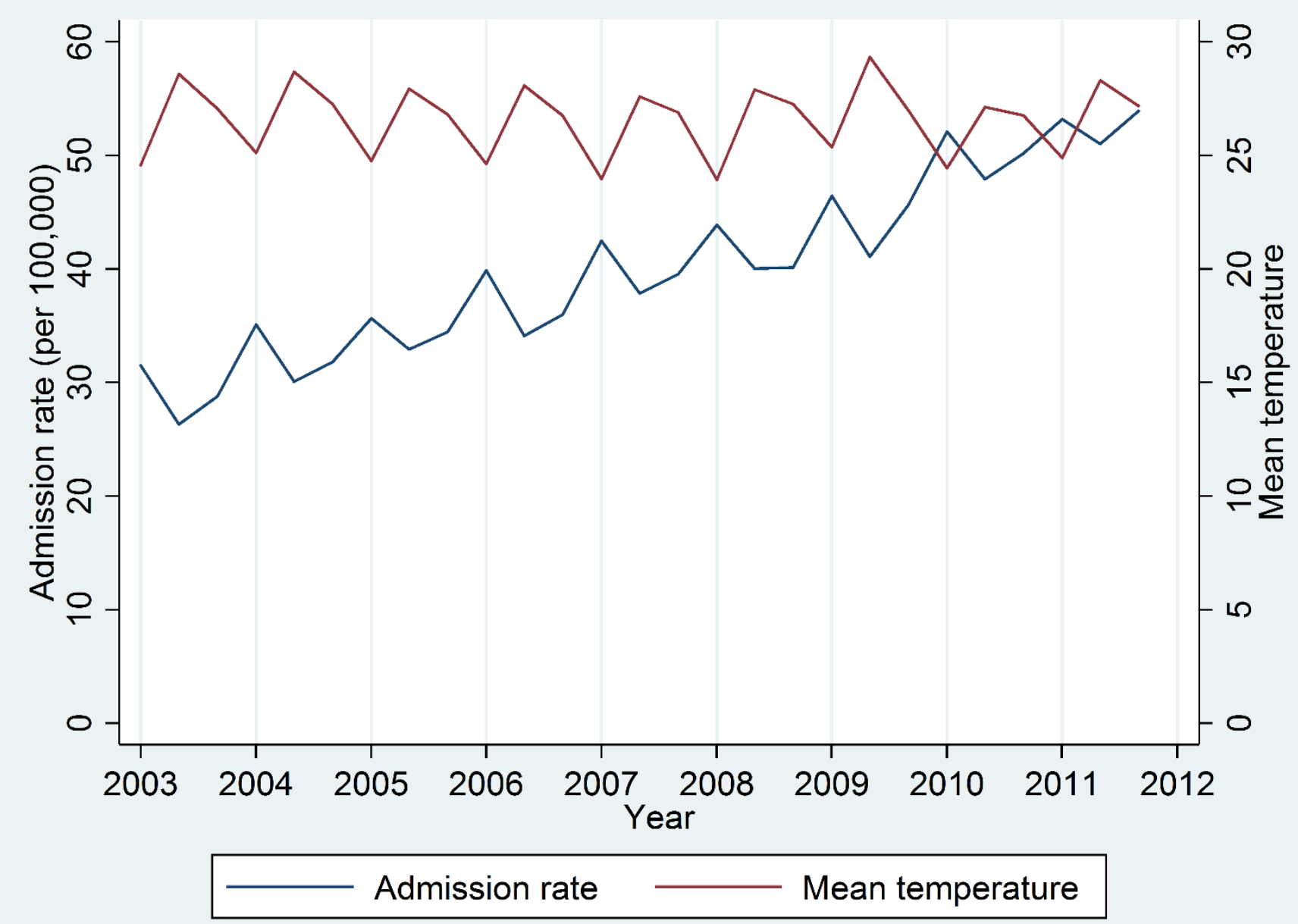


Figure 2. Incident stroke rate by season ( $($ rate/100,000)) and mean seasonal temperature (oC) (winter season 2003/4 - rainy season 2012).

Winter data for each year falls within vertical lines. 